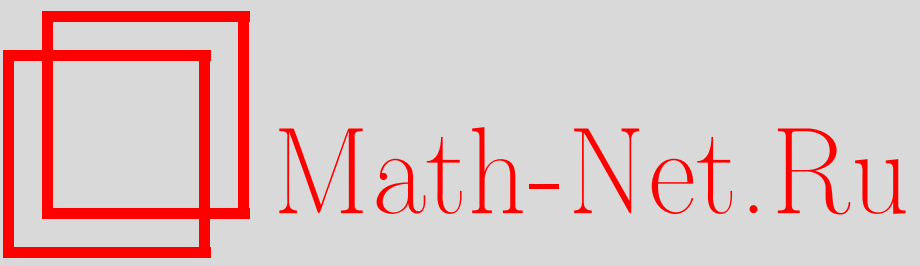

В. Г. Смирнов, Слои конечного автомата, Матем. вопр. криптогр., 2011, том 2, выпуск 1,97-117

DOI: https://doi.org/10.4213/mvk27

Использование Общероссийского математического портала Math-Net.Ru подразумевает, что вы прочитали и согласны с пользовательским соглашением

http://www.mathnet.ru/rus/agreement

Параметры загрузки:

IP : 54.205 .225 .156

26 апреля 2023 г., 14:25:01 


\author{
МАТЕМАТИЧЕСКИЕ ВОПРОСЫ КРИПТОГРАФИИ \\ 2011 T. 2 № 1 C. 97-118
}

УДК 519.16

\title{
Слои конечного автомата
}

\author{
В. Г. Смирнов
}

Академия криптографии Российской Федерации, Москва

Получено 22.IV.2010

В работе определены слои автомата, изучены их свойства и получены условия принадлежности состояний автомата его слоям. Введено понятие $t$-развертки графа инициального автомата как ориентированного графа с помеченными ребрами, с помощью которого задача перечисления прообразов отрезка выходной последовательности длины $t$ сводится к построению графа решений системы уравнений $k$-значной логики. Предложен алгоритм построения графа решений указанной системы, трудоемкость которого пропорциональна числу вершин $t$-развертки.

Показано, что трудоемкость алгоритма может как полиноминально, так и экспоненциально зависеть от $t$.

Ключевые слова: конечные автоматы, полугруппы преобразований, граф переходов

\section{Layers of a finite automaton}

\section{G. Smirnov \\ Academy of Cryptography of Russian Federation, Moscow}

\begin{abstract}
Layers of automaton are defined, their properties are investigated and conditions for a state of automata to belong to a layer are found. A notion of $t$-unrollment of initial automaton graph is introduced as an oriented graph with marked edges; this notion is used for reduction the enumeration of preimages of the output sequence segment to the construction of graph of solutions for a system of $k$-valued logic equations. An algorithm for the construction of such graph with complexity proportional to the number of vertices of $t$-unrollment is designed. The complexity may depend on $t$ polynomially or exponentially.
\end{abstract}

Key words: finite automata, semigroups of transforms, transition graph

Citation: Mathematical Aspects of Cryptography, 2011, vol. 2, no. 1, pp. 97-118 (Russian). 


\section{§ 1. Введение}

Задачи анализа конечного автомата приводят к необходимости изучения различных свойств его выходных последовательностей, последовательностей результирующих преобразований и состояний. При этом практический интерес представляет рассмотрение как регулярных, так и нерегулярных автоматов.

В увязке с анализом регулярных автоматов в [1] были исследованы такие параметры и объекты как слой, глубина и ширина группы относительно фиксированного порождающего множества.

Аналогичные понятия могут быть введены в рассмотрение и для состояний конечного, вообще говоря, нерегулярного автомата.

Далее рассматривается конечный автомат $\boldsymbol{A}=(X, A, Y, q(x, a), h(x, a))$,

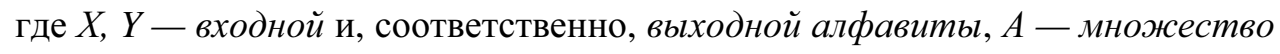
состояний, функиия переходов $q(x, a)$ определена на множестве $X \times A$ и принимает значения из множества $A$, функция выходов $h(x, a)$ определена на множестве $X \times A$ и принимает значения из множества $Y$.

Через $\boldsymbol{A}\left(a_{0}\right)=\left(X, A, a_{0}, Y, q(x, a), h(x, a)\right)$ обозначен инициальный автомат, получающийся из автомата $\boldsymbol{A}$ при фиксации начального состояния $a_{0} \in A$.

Введём обозначения:

$Z$ - кольцо целых чисел,

$X=Y=Z_{k} \subset Z$, где $Z_{k}=\{0,1, \ldots, k-1\}, k>1$.

Пусть $q_{x}: a \rightarrow q(x, a)$ - преобразование множества $A$, задаваемое функцией $q(x, a)$ при фиксированном значении $x$.

Определим операцию умножения на множестве $Q=\left\{q_{x} \mid x \in X\right\}$, положив $q_{y} \cdot q_{x}: a \rightarrow q(x, q(y, a))$ для всех $x, y \in Z_{k}$. Относительно введённой операции множество $Q$ порождает полугруппу $F_{A}$ результирующих преобразований множества состояний автомата $\boldsymbol{A}$, которую далее будем называть полугруппой автомата $\boldsymbol{A}$. Элементы полугруппы $F_{\boldsymbol{A}}$ будем рассматривать как функции одного переменного, тогда операцией полугруппы служит суперпозиция функций, задаваемая соотношением:

$$
(g \cdot f)(a)=g(f(a)), f, g \in F_{A} .
$$

Положим $a \tau b$, если существуют такая последовательность состояний $a=a_{1}, \ldots, a_{s}=b$ автомата $\boldsymbol{A}$ и такая последовательность знаков входного алфавита $x_{1}, \ldots, x_{s-1}$, что для каждой пары $a_{i}, a_{i+1}, i=1, \ldots, s-1$, либо 
$q_{x_{i}}\left(a_{i}\right)=a_{i+1}$, либо $q_{x_{i}}\left(a_{i+1}\right)=a_{i}$. Отношение $\tau$, являющееся эквивалентностью, называют отношением связности. Если число классов эквивалентности отношения связности равно единице, то автомат называют связным.

Из определения инициального автомата следует, что автомат $\boldsymbol{A}\left(a_{0}\right)$ является связным.

Графом переходов автомата $\boldsymbol{A}$ называют ориентированный помеченный граф $G_{A}$ с множеством вершин $A$, в котором тогда и только тогда имеется ребро из $a$ в $b$, помеченное меткой $x$, когда существует такое преобразование $q_{x} \in Q$, что $q_{x}(a)=b$.

Графом переходов инициального автомата $\boldsymbol{A}\left(a_{0}\right)$ называют подграф орграфа $G_{A}$, задаваемый вершиной $a_{0}$ и множеством $F_{A}\left(a_{0}\right)$ достижимых из нее вершин.

Некоторые из рассматриваемых определений и утверждений о свойствах конечного автомата имеют аналоги на языке теории графов, на что указывают соответствующие ссылки.

Приведём краткое изложение содержания статьи.

В § 2 рассматриваются инвариантные множества и компоненты сильной связности автомата. Вводится отношение орбитной эквивалентности на множестве состояний автомата, определяется цикловой индекс для компонент сильной связности, изучаются его свойства.

В § 3 даются определения слоев и оболочки автомата, модуля однозначности состояний инициального автомата. Получены оценки глубины и периода слоёв автомата. В теореме 2.6 сформулированы условия принадлежности состояний автомата его слоям. Приводится интерпретация полученных результатов для случая регулярного автомата.

В $§ 4$ вводится в рассмотрение $t$-развертка графа автомата, являющаяся $\Delta$-графом в смысле статьи [6]. Вершины $t$-развертки уровня $i$ совпадают с $i$-м слоем автомата $\boldsymbol{A}, i=1, \ldots, t$. Показано, что задача перечисления множества возможных входных последовательностей инициального автомата $\boldsymbol{A}\left(a_{0}\right)$ по выходу длины $t$ сводится к построению графа решений некоторой системы уравнений $k$-значной логики. Предложен алгоритм построения графа решений указанной системы, трудоемкость которого пропорциональна числу вершин $t$-развертки автоматного графа.

Рассмотрены примеры, показывающие, что трудоемкость алгоритма может зависеть от $t$ как полиноминально, так и экспоненциально.

Отмечено также, что применение более трудоемкого алгоритма из [8] позволяет построить граф решений системы по искаженной выходной последовательности. 


\section{§ 2. Инвариантные множества состояний и компоненты сильной связности автомата}

2.1. Инвариантные множества состояний. Подмножество $N \subseteq A$ называют инвариантным множеством состояний автомата $\boldsymbol{A}$, если $F_{A}(N) \subseteq N$.

Очевидно, что объединение и пересечение инвариантных множеств состояний - снова инвариантные множества.

Для любого подмножества $B \subseteq A$ множество

$$
\operatorname{St}(B)=\left\{f \in F_{A}, f(B)=B\right\}
$$

называют $B$-стабилизатором полугруппы $F_{A}$. При $B=\{a\}$ множество $\operatorname{St}(a)$ называют стабилизатором состояния а полугруппы $F_{A}$.

Непустое подмножество $G$ элементов полугруппы $F$ называется нормальной подполугруппой, если существует гомоморфизм $\varphi$ полугруппы $F$ на полугруппу с единицей $S$, при котором множество $G$ является полным прообразом единицы [4].

Теорема 2.1. Если $N$ - инвариантное множество состояний автомата $\boldsymbol{A}$ и $\operatorname{St}(N) \neq \varnothing$, то $\operatorname{St}(N)$ является нормальной подполугруппой полугруп$n b l F_{A}$.

Доказательство. Введём на полугруппе $F_{A}$ отношение эквивалентности $\eta_{N}$, положив $f \eta_{N} g$ тогда и только тогда, когда для любой функции $h \in F_{A}$ соотношения $h \cdot f \in \mathrm{St}(N), h \cdot g \in \mathrm{St}(N)$ выполняются только одновременно. Рассмотрим имеющиеся возможности.

1) Если $f, g \notin \operatorname{St}(N)$, то $f(N) \cap N \subset N, g(N) \cap N \subset N$, следовательно, при любой функции $h \in F_{A}$ имеем $h \cdot f, h \cdot g \cdot \notin \operatorname{St}(N)$.

2) Если $f, g \in \operatorname{St}(N)$, то при любой функции $h \in F_{A}$ имеем $h(f(N))=$ $=h(g(N))$, и соотношения $h \cdot f \in \mathrm{St}(N), h \cdot g \in \mathrm{St}(N)$ выполняются только одновременно.

3) Если $f \in \operatorname{St}(N), g \notin \operatorname{St}(N)$, то, положив $h=f$, получим $f \cdot f \in \operatorname{St}(N)$, $f \cdot g \notin \operatorname{St}(N)$, и $f \not \chi_{N} g$.

Следовательно, $f \eta_{N} g$ тогда и только тогда, когда или $f \notin \operatorname{St}(N)$, $g \notin \operatorname{St}(N)$, или $f \in \operatorname{St}(N), g \in \operatorname{St}(N)$.

Очевидно, что эквивалентность $\eta_{N}$ является конгруэнцией, содержащей либо два класса, если $F_{A} \neq \mathrm{St}(N)$, либо один класс, если $F_{A}=\operatorname{St}(N)$. В первом случае $F_{A} / \eta_{N} \cong S^{(0)}$, где $S^{(0)}-$ полугруппа, состоящая только из нуля и единицы. Второй случай тривиален, так как полугруппа $F_{A} / \eta_{N}$ состоит из одного элемента. Полугруппа $\operatorname{St}(N)$ оказывается во всех случаях полным прообразом единицы, т. е. нормальной подполугруппой. Теорема доказана. 
2.2. Орбиты и орбитная эквивалентность. Минимальное инвариантное множество $O(a)$, содержащее состояние $a \in A$, назовем орбитой состояния $а$.

Из определения орбиты следует, что $O(a)=a \cup F_{A}(a)$.

Любое объединение орбит является инвариантным множеством. Верно и обратное: что любое инвариантное множество является объединением орбит.

Очевидно, автомат $\boldsymbol{A}\left(a_{0}\right)$ совпадает с ограничением автомата $\boldsymbol{A}$ на множество $O\left(a_{0}\right)$.

Определение 2.1. Состояния $a, b \in A$ назовем орбитно эквивалентными $(a \underset{O}{\sim} b)$, если $O(a)=O(b)$.

Отношение $\sim$ является отношением эквивалентности, легко проверить его рефлексивность, симметричность и транзитивность. Классы орбитной эквивалентности назовем $O$-классами.

Обозначим через $F(a, b)$ множество функций $f \in F_{A}$, удовлетворяющих условию $f(a)=b$. В частности, $\operatorname{St}(a)=F(a, a)$.

Если $F(a, b) \neq \varnothing$, то положим $b<_{d} a$. В этом случае говорят, что состояние $b$ достижимо из $a$ [5].

Если $b<_{d} a$ и $a<_{d} b$, то состояния $a, b$ называют взаимно связанными.

Следующие утверждения вытекают непосредственно из определения орбиты и орбитной эквивалентности.

1) $O(b) \subseteq O(a)$ тогда и только тогда, когда $b=a$ либо $b<_{d} a$.

2) $O(b)=O(a)$ тогда и только тогда, когда $b=a$ либо состояния $a, b$ взаимно связаны.

3) $O(a)=F_{A}(a)$ тогда и только тогда, когда $\operatorname{St}(a) \neq \varnothing$.

Введём обозначение $O_{a}$ для множества состояний, орбитно эквивалентных состоянию $a$.

Лемма 2.1. Если $\left|O_{a}\right|>1$, то $F(a, a) \neq \varnothing$.

Доказательство. Пусть $b \in O_{a}, b \neq a$. Тогда $F(b, a) \neq \varnothing, F(a, b) \neq \varnothing$, откуда вытекает утверждение леммы.

Следуя известному определению [например, 4], функции $f, g \in F_{A}$ назовём $R$-эквивалентными ( $\underset{R}{\sim} g$ ), если они порождают один и тот же главный правый идеал полугруппы $F_{A}$, т. е. если $F_{A} \cdot f \cup f=F_{A} \cdot g \cup g$.

Лемма 2.2. Из $f, g \in F_{A}$ u $\underset{R}{\sim} g$ следует, что $O(f(a))=O(g(a))$ для любого $a \in A$.

Доказательство. Из условий леммы вытекает существование таких функций $h, h^{\prime} \in F_{A}$, что $h \cdot f=g, h^{\prime} \cdot g=f$, откуда для любого $a \in A$ получаем $h(f(a))=g(a), h^{\prime}(g(a))=f(a)$, что доказывает утверждение леммы. 
Лемма 2.3. Пусть $\underset{O}{a} \underset{\sim}{\sim}$ u $f(a)=b$, где $f=q_{j_{k}} \cdot \ldots \cdot q_{j_{1}}$. Тогдa $q_{j_{m}} \cdot \ldots \cdot q_{j_{1}}(a) \underset{o}{\sim} b$ для любого $m=1, \ldots k$.

Доказательство. Невыполнение утверждения леммы ведёт к противоречию с условием $a \sim$ $b$.

Назовем класс $O_{a}$ максимальным $O$-классом (минимальным $O$-классом), если не существует такого класса $O_{b}$, что $O_{b} \supset O_{a}\left(O_{b} \subset O_{a}\right)$.

Введем обозначения $O_{1}^{(\min )}, \ldots, O_{s}^{(\min )}$ для минимальных $O$-классов, $O_{1}^{(\max )}, \ldots, O_{t}^{(\max )}$ для максимальных $O$-классов и положим

$$
O^{(\min )}(\boldsymbol{A})=\cup_{j=1}^{s} O_{j}^{(\min )}, O^{(\max )}(\boldsymbol{A})=\cup_{j=1}^{t} O_{j}^{(\max )} .
$$

Для инициального автомата $\boldsymbol{A}\left(a_{0}\right)$ имеем $O^{(\max )}\left(\boldsymbol{A}\left(a_{0}\right)\right)=O\left(a_{0}\right)$, $O^{(\min )}\left(\boldsymbol{A}\left(a_{0}\right)\right)=O^{(\min )}(\boldsymbol{A}) \cap O\left(a_{0}\right)$.

Напомним, что минимальный двусторонний идеал полугруппы называют её ядром. Ядро полугруппы $F_{A}$ обозначим $K_{F_{A}}$.

Лемма 2.4. Имеют место соотношения:

1) $O_{a}=O(a)$ тогда и только тогда, когда $a \in O^{(\min )}(A)$;

2) $O^{(\min )}(\boldsymbol{A})=K_{F_{A}}(A)$.

Доказательство. Если $a \notin O^{(\min )}(\boldsymbol{A})$, то существует такое состояние $b \in O(a)$, что $O(b) \subset O(a)$, следовательно, $O_{a} \neq O(a)$. Если $a \in O^{(\min )}(\boldsymbol{A})$, то не существует орбиты $O(b) \subset O(a)$, следовательно, $O_{a}=O(a)$. Первое утверждение доказано.

Если $f \in K_{F_{A}}$, то из свойств ядра полугруппы преобразований следует, что $f(A)=O_{j}^{(\min )}$ для некоторого $j \in\{1, \ldots, s\}$. Обратно для каждого $j \in\{1, \ldots, s\}$ найдётся функция $f \in K_{F_{A}}$, для которой $f(A)=O_{j}^{(\min )}$. Следовательно, $K_{F_{A}}(A)=O^{(\min )}(\boldsymbol{A})$. Лемма доказана.

2.3. Порождающие множества состояний. Для любого множества $B \subseteq A$ положим $O(B)=\cup_{b \in B} O(b)$.

Множество $B$, для которого $O(B)=A$, называют порождающим множеством состояний автомата $\boldsymbol{A}$. Порождающее множество называется минимальным, если оно не содержит собственного подмножества, являющегося порождающим множеством [5].

Напомним, что подмножество $T$ множества $M$, содержащее в точности один элемент из каждого класса эквивалентности $\mathcal{E}$ на $M$, называется трансверсалью множества М по модулю $\varepsilon$ [2]. 
Теорема 2.2. Множество $B \subseteq A$ тогда и только тогда является минимальным порождающим множеством состояний автомата $A$, когда $B$ является трансверсалью классов орбитальной эквивалентности, принадлежаших множеству $O^{(\max )}(\boldsymbol{A})$.

Доказательство. Классы ограничения отношения эквивалентности $\tilde{o}$ на множество $O^{(\max )}(\boldsymbol{A})$ перечислены в разбиении $O^{(\max )}(\boldsymbol{A})=\bigcup_{j=1}^{t} O_{j}^{(\max )}$. УТверждение теоремы следует из определения орбитальной эквивалентности.

2.4. Компоненты сильной связности автомата. Каждое максимальное подмножество состояний автомата, в котором любые два состояния взаимно связаны, называют компонентой сильной связности.

Лемма 2.5. Множество $B \subseteq A$ тогда и только тогда является компонентой сильной связности, когда $\operatorname{St}(b) \neq \varnothing$ и $B=O_{b}$ для любого $b \in B$.

Доказательство. Из определения 2.1 следует, что при $\operatorname{St}(b) \neq \varnothing$ множество $O_{b}$ является максимальным множеством, в котором все состояния взаимно связаны, т. е. компонентой сильной связности. Обратно, пусть $B-$ компонента сильной связности. Тогда для любого $b \in B$ имеем $\operatorname{St}(b) \neq \varnothing$, и если $a \in B$, то $O(a)=O(b)$, а в случае $a \notin B$ имеем $O(a) \neq O(b)$. Следовательно, $B=O_{b}$. Лемма доказана.

Обозначим через $C(A)$ множество состояний, являющееся объединением компонент сильной связности автомата. Соответственно, объединение компонент сильной связности автомата $\boldsymbol{A}\left(a_{0}\right)$ обозначим $C\left(O\left(a_{0}\right)\right)$. Очевидно, что $C(A) \cap O\left(a_{0}\right)=C\left(O\left(a_{0}\right)\right)$.

Из леммы 2.5 следует, что

$$
C(A)=\underset{a, \mathrm{St}(a) \neq \varnothing}{\cup} O(a)=\{a \in A \mid \operatorname{St}(a) \neq \varnothing\} .
$$

В конечной полугруппе некоторая степень любого элемента $f$ является идемпотентом, для которого используется обозначение $e_{f}$. Множество всех идемпотентов полугруппы $F$ обозначим $E(F)$.

Если $f \in \operatorname{St}(a)$, то из определения идемпотента $e_{f}$ следует, что $e_{f}(a)=a$. Отсюда вытекает соотношение

$$
C(A)=\underset{e \in E(F)}{\cup} e(A) .
$$

Из лемм 2.1, 2.5 следует, что множество $A \backslash C(A)$ состоит из состояний $b$, для которых $\left|O_{b}\right|=1, \operatorname{St}(a)=\varnothing$.

Состояния $\mathrm{a} \in \mathrm{C}(\mathrm{A})$ назовём цикловыми состояниями.

2.5. Цикловые индексы цикловых состояний автомата. Если $q_{x_{n}} \cdot q_{x_{n-1}} \cdot \ldots \cdot q_{x_{1}}=f$, то будем говорить, что слово $q_{x_{n}} \ldots q_{x_{1}}$ является записью длины $n$ (в алфавите $Q$ ) функции $f \in F_{A}$. 
Функции $f \in F_{A}$ поставим в соответствие множество натуральных чисел $\Lambda(f)$, состоящее из длин записей функции $f$ в алфавите $Q$.

Состоянию $a \in C(A)$ поставим в соответствие множество натуральных чисел

$$
Z(a)=\underset{f \in \operatorname{St}(a)}{\cup} \Lambda(f) .
$$

Определение 2.2. Цикловым индексом состояния $a \in C(A)$ назовём число $\omega(a)=$ НОД $\{z \mid z \in Z(a)\}$.

Множество $Z(a)$ замкнуто относительно сложения и, следовательно, содержит все числа, кратные $\omega(a)$, кроме, возможно, их конечного подмножества.

Лемма 2.6. Если $a \in C(A)$ u $\underset{o}{\sim} b$, mо $\omega(a)=\omega(b)$.

Доказательство. Если $\left|O_{a}\right|=1$, то утверждение леммы тривиально. Пусть $\left|O_{a}\right|>1$ и $a \neq b$. Так как $a \sim b$, то найдутся такие функции $f, g \in S$, что $f(a)=b, g(b)=a$. Тогда

откуда получаем:

$$
g \cdot \operatorname{St}(b) \cdot f \subseteq \operatorname{St}(a), \quad f \cdot \operatorname{St}(a) \cdot g \subseteq \operatorname{St}(b),
$$

$$
Z(a) \supseteq\{\Lambda(g \cdot f), Z(b)+\Lambda(g \cdot f)\}, Z(b) \supseteq\{\Lambda(f \cdot g), Z(a)+\Lambda(f \cdot g)\} .
$$

Следовательно, $\omega(a) \mid \omega(b)$ и $\omega(b) \mid \omega(a)$, откуда вытекает справедливость утверждения леммы.

\section{§ 3. Слои и оболочка автомата}

3.1. Определение слоёв автомата. В мультипликативной полугруппе всех подмножеств полугруппы $F_{A}$ множество $Q$ порождает циклическую подполугруппу

$$
<Q>=\left\{Q, Q^{2}, \ldots, Q^{\rho}, \ldots, Q^{\rho+\omega-1}\right\},
$$

где $Q^{\rho}=Q^{\rho+\omega}-$ первое повторение в последовательности степеней множества $Q$ [3]. Таким образом,

$$
F_{A}=\stackrel{\rho+\omega-1}{\bigcup} Q^{i}
$$

Из определения умножения подмножеств следует, что множество $Q^{i}$, $1 \leq i \leq \rho+\omega-1$, состоит из всех элементов полугруппы, записываемых хотя бы одним способом словом длины $i$ в алфавите $Q$.

В соответствии с определениями из [2], числа $\rho$ и $\omega$ назовём цииклической глубиной и периодом циклической полугруппы $<Q>$.

Автомату $\boldsymbol{A}\left(a_{0}\right)$ и входной последовательности $x_{1}, \ldots, x_{t}$ соответствуют три последовательности:

1) последовательность результирующчих преобразований

$$
f_{1}=q_{x_{1}}, f_{2}=q_{x_{2}} \cdot q_{x_{1}}, \ldots, f_{t}=q_{x_{t}} \cdot q_{x_{t-1}} \cdot \ldots \cdot q_{x_{1}} ;
$$


2) последовательность состояний автомата $\boldsymbol{A}\left(a_{0}\right)$

$$
a_{0}, a_{1}=q_{x_{1}}\left(a_{0}\right), \ldots, a_{t}=q_{x_{t}}\left(a_{t-1}\right)
$$

3) выходная последовательность автомата $A\left(a_{0}\right)$

$$
y_{1}=h\left(a_{0}, x_{1}\right), y_{2}=h\left(a_{1}, x_{2}\right), \ldots, y_{t}=h\left(a_{t}, x_{t-1}\right) .
$$

Для $G \subseteq F_{A}, B \subseteq A$ положим $G(B)=\underset{g \in G \in B}{\cup} \cup(b)$.

Последовательности

$$
Q^{j}(A), Q^{j}\left(a_{0}\right), \quad j=1,2, \ldots,
$$

являются смешанными периодическими последовательностями. Очевидно, что

$$
Q^{j}(A)=\underset{a_{0} \in A}{\cup} Q^{j}\left(a_{0}\right) .
$$

Введем обозначения $\rho(A), \rho\left(O\left(a_{0}\right)\right)$ и $\omega(A), \omega\left(O\left(a_{0}\right)\right)$ для величин, определяемых первым повторением $Q^{\rho(A)}(A)=Q^{\rho(A)+\omega(A)}(A)$, соответственно, $Q^{\rho\left(a_{0}\right)}\left(a_{0}\right)=Q^{\rho\left(a_{0}\right)+\omega\left(O\left(a_{0}\right)\right.}\left(a_{0}\right)$ в последовательностях (3.5).

Определение 3.1. 1) Для $j=1, \ldots, \rho(A)+\omega(A)-1$ назовем $j$-м слоем автомата $\boldsymbol{A}$ множество $Q^{j}(A)$.

2) Для $j=1, \ldots, \rho\left(O\left(a_{0}\right)\right)+\omega\left(O\left(a_{0}\right)\right)-1$ назовем $j$-м слоем инициального автомата $O\left(a_{0}\right)$ множество $Q^{j}\left(a_{0}\right)$.

3) Назовем число $\rho(A)$ глубиной, а число $\omega(A)-$ периодом слоев автомama $A$.

4) Назовем число $\rho\left(O\left(a_{0}\right)\right)$ глубиной, а число $\omega\left(O\left(a_{0}\right)\right)-$ периодом слоев автомата $\boldsymbol{A}\left(a_{0}\right)$.

3.2. Оболочка автомата. Для каждого состояния $a \in A$ определим множество $O^{*}(a)$, положив

$O^{*}(a)=\left\{b \in A \mid\right.$ существует такая функция $f \in F_{A}$, что $\left.f(b)=a\right\}$. из $a$.

Состояния $b \in O^{*}(a)$ назовем, аналогично [5], обратно достижимыми

Очевидно, что из $a \underset{o}{\sim} b$ следует, что $O^{*}(a)=O^{*}(b)$, и $a \in O^{*}(a)$ тогда и только тогда, когда $a \in C(a)$.

Определение 3.2. Оболочкой множества состояний автомата А назовём множество

$$
W(A)=\left\{a \mid \text { из } b \in O^{*}(a) \text { следует, что } \operatorname{St}(b)=\varnothing\right\} .
$$

Данное определение допускает эквивалентную формулировку: $a \in W(A)$ тогда и только тогда, когда не существует состояния $b \in C(A), \mathrm{O}(b) \supseteq \mathrm{O}(a)$.

Теорема 3.1. Имеет место соотношение

$$
W(A) \cap\left(\bigcup_{j=\rho(A)}^{\rho(A)+\omega(A)-1} Q^{j}(A)\right)=\varnothing .
$$


Доказательство. Пусть $a \in Q^{j}(A), \rho(A) \leq j \leq \rho(A)+\omega(A)-1$. Из свойств циклической полугруппы $\langle Q>$ следует, что в этом случае найдутся такое состояние $b \in O^{*}(a)$ и такое преобразование $f=q_{x_{t}} \cdot q_{x_{t-1}} \cdot \ldots \cdot q_{x_{1}}$, где $t>|A|$, что $f(b)=a$. Обозначим $f^{(j)}=q_{x_{j}} \cdot q_{x_{j-1}} \cdot \ldots \cdot q_{x_{1}}, j=1, \ldots, t$. В последовательности $f^{(j)}(b)$, в силу условия $t>|A|$, найдутся повторения, например, $f^{(s)}(b)=f^{(r)}(b), s<r$. Тогда $f^{(s)}(b) \in C(A)$, откуда следует, что $a \notin W(A)$. Теорема доказана.

Следствие 3.1. Если $t \geq \rho(A)+\omega(A)-1$, то

$$
\bigcup_{j=\rho(A)}^{t} Q^{j}(A)=A \backslash W(A) .
$$

Справедливость этого утверждения вытекает непосредственно из определения 3.2 и теоремы 3.1.

Пусть $W(A) \neq \varnothing$. Из $a \in W(A)$ вытекает, что $O_{a}=\{a\}$. Зададим частичный порядок на множестве $W(A)$, положив $a>b$ тогда и только тогда, когда $O(a) \supset O(b)$. Обозначим через $W^{(1)}=W^{(\max )}(A)$ множество максимальных элементов в $W(A)$, через $W^{(2)}-$ множество максимальных элементов в $W(A) \backslash W^{(1)}$, если $W(A) \backslash W^{(1)} \neq \varnothing$ и т. д. На некотором шаге $r(A) \geq 1$ получим непустое множество

$$
W^{(r(A))}=W(A) \backslash \bigcup_{j=1}^{r(A)-1} W^{(j)},
$$

не содержащее сравнимых элементов. Тогда

$$
W(A)=\bigcup_{j=1}^{r(A)} W^{(j)} .
$$

Если $W(A)=\varnothing$, то положим $r(A)=0$.

Лемма 3.1. Множество $W^{(\max )}(A)$ содержит все состояния, не принадлежащие ни одному из слоев автомата.

Действительно, $a \in W^{(\max )}(A)$ тогда и только тогда, когда $O^{*}(a)=\varnothing$, т. е. $a \notin F_{A}(A)$. Справедливость утверждения леммы вытекает из определения слоев автомата.

Для $a \in A$ положим

$$
L(a)=\underset{b \in W^{(\max )}(A)}{\cup} \underset{f \in F(b, a)}{\cup} \Lambda(f) .
$$

Из теоремы 3.1 и леммы 3.1 следует, что $L(a)=\varnothing$ при $a \in W^{(1)}(A)$ и множество $L(a)$ конечно при $a \in W(A) \backslash W^{(1)}(A)$. Во всех других случаях множество $L(a)$ счётное.

Для случая $a \in W(A) \backslash W^{(1)}(A)$ введем обозначение

$$
l(a)=\max \{l \mid l \in L(a)\} .
$$

Если $a \in W^{(1)}(A)$, то положим $l(a)=0$. 

$=i-1$.

Теорема 3.2. Тогда и только тогда $a \in W^{(i)}, i=1, \ldots, r(A)$, когда $l(a)=$

Доказательство. Для $a \in W^{(1)}$ утверждение теоремы тривиально. Из определения множеств $W^{(i)}, i=1, \ldots, r(A)$, следует, что $W^{(i)} \subseteq Q\left(W^{(i-1)}\right)$. Поэтому из справедливости утверждения теоремы для $a \in Q\left(W^{(i-1)}\right)$ вытекает его справедливость для $a \in W^{(i)}$. Теорема доказана.

Оболочку автомата $\boldsymbol{A}\left(a_{0}\right)$ обозначим $W\left(O\left(a_{0}\right)\right)$.

Полученные результаты верны для автомата $\boldsymbol{A}\left(a_{0}\right)$. В этом случае $W\left(O\left(a_{0}\right)\right) \neq \varnothing$, только если $a_{0} \notin C(A)$, и тогда

$$
W^{(\max )}\left(O\left(a_{0}\right)\right)=\left\{a_{0}\right\} .
$$

При $a \neq a_{0}$ по определению инициального автомата имеем $F\left(a_{0}, a\right) \neq \varnothing$, следовательно, $L(a) \neq \varnothing$ и

$$
L(a)=\underset{f \in F\left(a_{0}, a\right)}{\cup} \Lambda(f) .
$$

\section{3. Значение периода и оценка глубины слоёв автомата.}

Теорема 3.3. 1) Для периода слоев автомата А верно равенство

$$
\omega(A)=\operatorname{HOK}\{\omega(a), a \in C(A)\} .
$$

2) Для глубины слоев автомата А верна оценка

$$
\rho(A) \geq r(A) .
$$

Доказательство. Обозначим $\tilde{\omega}=\operatorname{HOK}\{\omega(a), a \in C(A)\}$. Если $a \in C(A)$, то из $a \in Q^{s}(A)$ следует, что $a \in Q^{s+a(a)}(A)$. Следовательно, для любого $s \geq \rho(A)$ и всех состояний $a \in Q^{s}(A) \cap C(A)$ имеем $\omega(a) \mid \omega(A)$, откуда $Q^{s}(C(A))=Q^{s+\tilde{\omega}}(C(A))$ и $\tilde{\omega} \mid \omega(A)$.

Покажем, что для $s \geq \rho(A)$ выполняется равенство $Q^{s}(A)=Q^{s+\tilde{\omega}}(A)$.

Если $a \in W(A)$, то согласно лемме 3.1 для $s \geq \rho(A)$ имеем $a \notin Q^{s}(A)$.

При $a \in C(A)$ равенство $Q^{s}(C(A))=Q^{s+\tilde{\omega}}(C(A))$ уже установлено.

При $a \in A \backslash(C(A) \cup W(A))$ имеем $O^{*}(a) \cap C(A) \neq \varnothing$, и если $a \in Q^{s}(A)$, то найдется такое состояние $b \in O^{*}(a) \cap C(A)$, что $a \in Q^{s+\omega(b)}(A)$, следовательно, $Q^{s}(A \backslash(C(A) \cup W(A)))=Q^{s+\tilde{\omega}}(A \backslash(C(A) \cup W(A)))$. Окончательно, $Q^{s}(A)=$ $=Q^{s+\tilde{\omega}}(A)$, откуда и $\omega(A)=\tilde{\omega}$. Первое утверждение теоремы доказано.

Из теоремы 3.2 вытекает, что $Q^{s}(A) \cap W(A) \neq \varnothing$ при $s<r(A)$, а из теоремы $3.1-$ что $Q^{s}(A) \cap W(A)=\varnothing$ при $s \geq r(A)$. Отсюда следует справедливость второго утверждения теоремы. Теорема доказана.

Для автомата $\boldsymbol{A}\left(a_{0}\right)$ справедливы аналогичные результаты.

Следствие 3.2. 1) Для периода слоёв автомата $А\left(a_{0}\right)$ верно равенство

$$
\omega\left(O\left(a_{0}\right)\right)=\operatorname{HOK}\left\{\omega(a), a \in C\left(O\left(a_{0}\right)\right)\right. \text {. }
$$

2) Для глубины слоёв автомата А( $\left.a_{0}\right)$ верна оценка

$$
\left.\rho\left(O\left(a_{0}\right)\right)\right\} \geq r\left(O\left(a_{0}\right)\right) \text {. }
$$




\section{4. Модуль однозначности состояний инициального автомата.}

Определение 3.3. Множество $L$ натуральных чисел, $|L|>1$, принадлежит вычету $\mu(\bmod \theta)$, где $0 \leq \mu \leq \theta-1$, если все числа $l \in L$ удовлетворяют сравнению $l \equiv \mu(\bmod \theta)$.

Положим

$$
\theta(L)=\text { НОД }\left\{\left|l_{1}-l_{2}\right|: l_{1}, l_{2} \in L, \quad l_{1} \neq l_{2}\right\} .
$$

Из определения 3.3 следует, что множество $L$ принадлежит некоторому вычету $\mu(\bmod \theta)$ для всех делителей $\theta$ числа $\theta(L)$ и только для них.

Если $L^{(1)}, L^{(2)} L^{(3)}-$ множества натуральных чисел, то легко видеть, что из $L^{(1)} \supseteq L^{(2)}$ следует, что $\theta\left(L^{(1)}\right) \mid \theta\left(L^{(2)}\right)$, а из $L^{(1)}=L^{(2)}+L^{(3)}$ следует, что $\theta\left(L^{(1)}\right)\left|\theta\left(L^{(2)}\right), \theta\left(L^{(1)}\right)\right| \theta\left(L^{(3)}\right)$.

Вычет, которому по mod $\theta(L)$ принадлежат числа $l \in L$, обозначим $\mu(L)$.

В случае $|L|=1$ положим $\theta(L)=1, \mu(L)=0$.

Определение 3.4. Модулем однозначности состояния $a \in O\left(a_{0}\right)$, $O^{*}(a) \neq \varnothing$, назовем число $\delta(a)=\theta(L(a))$, а соответствующий вычет $\gamma(a)=$ $=\mu(L(a))$ назовём вычетом однозначности состояния $a$.

Равенство $O^{*}(a)=\varnothing$ выполняется для состояния $a_{0}$ только при условии $a_{0} \notin C(A)$. В этом случае $L\left(a_{0}\right)=\varnothing$. Если же $a_{0} \in C(A)$, то

$$
L\left(a_{0}\right)=Z\left(a_{0}\right), \delta\left(a_{0}\right)=\omega\left(a_{0}\right), v\left(a_{0}\right)=0 .
$$

Лемма 3.2. Если $a, b \in O\left(a_{0}\right), b<_{d} a$, то $\delta(b) \mid \delta(a)$.

Доказательство. Из условий леммы следует существование такой функции $f \in F_{A}$, что $f(a)=f(b)$. Для любой константы $s \in \Lambda(f)$ имеем

$$
L(b) \supseteq L(a)+s=\bigcup_{l \in L(a)}(l+s) ;
$$

отсюда вытекает справедливость утверждения леммы.

Следствие 3.3. Если $\underset{\sim}{\sim} \underset{\sim}{\sim}$, mo $\delta(a)=\delta(b)$.

Утверждение следствия вытекает из леммы 3.2 по соображениям симметрии.

Лемма 3.3. Если $a \in C(A)$ u $O(b) \subseteq O(a)$, mo $\delta(b) \mid \omega(a)$.

Из условия леммы следует, что $\operatorname{St}(a) \neq \varnothing$, поэтому справедливо включение

$$
L(b) \supseteq L(a)+Z(a),
$$

откуда вытекает утверждение леммы.

Следствие 3.4. Если $a \in O\left(a_{0}\right) \backslash W\left(O\left(a_{0}\right)\right)$, mo $\delta(a) \mid \omega\left(O\left(a_{0}\right)\right)$.

Утверждение следствия вытекает из леммы 3.3 и следствия 3.3 .

Состояния $b \in O_{a}$, для которых $v(b)=\mu, 0 \leq \mu \leq \delta(a)-1$, объединим в подмножество $O_{a}^{(\mu)}$, так что

$$
O_{a}=\bigcup_{\mu=0}^{\delta(a)-1} O_{a}^{(\mu)} .
$$


Лемма 3.4. Если $a \in C(A)$, то в разложении (3.8) нет пустых множеств.

Доказательство. Пусть $a \in C(A), f \in \operatorname{St}(a)$ и $f=q_{j_{k}} \cdot \ldots \cdot q_{j_{1}}$. По лемме 2.3 для любого $m=1, \ldots, k$ имеем $q_{j_{m}} \cdot \ldots \cdot q_{j_{1}}(a) \in O_{a}$. Следовательно,

$$
v\left(L\left(q_{j_{m}} \cdot \ldots \cdot q_{j_{1}}(a)\right)\right) \equiv v(L(a))+m(\bmod \delta(a)),
$$

откуда вытекает справедливость утверждения леммы.

3.5. Условия принадлежности слоям инициального автомата. В следующем утверждении даны условия принадлежности состояний $a \in O\left(a_{0}\right)$ слоям автомата $\boldsymbol{A}\left(a_{0}\right)$ в зависимости от значений введённых выше параметров.

Теорема 3.4. 1) Если $a \in W\left(O\left(a_{0}\right)\right)$, то $a \in Q^{j}\left(a_{0}\right)$ тогда и только тогда, когда $j \in L(a)$.

2) Если $a \in Q^{\mathrm{\rho}\left(O\left(a_{0}\right)\right)+j}\left(a_{0}\right), j=0,1, \ldots, \omega\left(O\left(a_{0}\right)\right)-1$, то выполняется сравнение $\rho\left(O\left(a_{0}\right)\right)+j \equiv v(a)(\bmod \delta(a))$.

3) Из условия $a \in C\left(O\left(a_{0}\right)\right) \cap Q^{\rho\left(O\left(a_{0}\right)\right)+j}\left(a_{0}\right), j=0,1, \ldots, \omega\left(O\left(a_{0}\right)\right)-1$, дополнительно следует, что для всех $s \equiv j(\bmod \omega(a))$ имеем $a \in$ $\in Q^{\rho\left(O\left(a_{0}\right)\right)+k}\left(O\left(a_{0}\right)\right)$.

4) Если $\delta(a)=\omega(a)$, то $a \in Q^{\rho\left(O\left(a_{0}\right)\right)+j}\left(a_{0}\right)$ тогда и только тогда, когда $\rho\left(O\left(a_{0}\right)\right)+j \equiv \psi(a)(\bmod \delta(a))$.

Доказательство. Утверждение 1) теоремы вытекает из определения множества $L(a)$.

2) Состояние $a \in Q^{\rho\left(O\left(a_{0}\right)\right)+j}\left(O\left(a_{0}\right)\right)$ тогда и только тогда, когда $\rho\left(a_{0}\right)+$ $+j \in L(a)$. Отсюда следует справедливость второго утверждения теоремы.

3) Если $l \in L(a)$, то $l+s \omega(a) \in L(a)$ для всех достаточно больших $s$. Отсюда следует справедливость третьего утверждения теоремы.

4) В случае $\delta(a)=\omega(a)$ последнее утверждение теоремы следует из второго и третьего утверждений.

3.6. Случай регулярного автомата. Автомат $\boldsymbol{A}$ называют регулярным, если полугруппа $F_{A}$ является группой. В случае регулярного автомата $W(A)=\varnothing, A=C(A)$, отношение орбитной эквивалентности совпадает с отношением взаимной достижимости, каждая орбита является компонентой сильной связности, и для всех состояний $a \in \boldsymbol{A}$ выполняется равенство $O_{a}=O(a)$. Все орбиты попарно не сравнимы, поэтому $O^{(\min )}(\boldsymbol{A})=O^{(\max )}(\boldsymbol{A})$.

Теорема 2.2 в случае регулярного автомата сводится к следующему утверждению. 
Теорема 2.2'. Множество $\mathrm{B} \subseteq \mathrm{A}$ тогда и только тогда является минимальным порождающим множеством состояний регулярного автомата $\mathbf{A}$, когда В является трансверсалью компонент сильной связности автомата $\mathbf{A}$.

Так как в случае регулярного автомата $A=C(A)$, то для всех состояний $a \in A$ выполняются равенства $\omega(a)=\delta(a)=\omega(A)$, и теорема 3.4 сводится к следующему утверждению.

Теорема 3.4'. Состояние а автомата $\boldsymbol{A}\left(a_{0}\right)$ тогда и только тогда принадлежит слою $Q^{\rho\left(0\left(a_{0}\right)\right)+j}\left(a_{0}\right)$, когда $\rho\left(O\left(a_{0}\right)\right)+j \equiv v(a)(\bmod \omega(a))$.

\section{§ 4. O $t$-развёртке графа инициального автомата}

4.1. Определение и основные свойства $t$-развертки графа автомата $\boldsymbol{A}\left(\boldsymbol{a}_{0}\right)$. Положим $Q^{0}\left(a_{0}\right)=a_{0}$.

Определение 4.1. Назовём t-разверткой графа переходов автомата $\boldsymbol{A}\left(a_{0}\right)$ граф $R\left(t ; a_{0}\right)$ с множеством вершин $V\left(t ; a_{0}\right)=\bigcup_{i=0}^{t} \bigcup_{a \in Q^{i}\left(a_{0}\right)}(a, i)$, в котором вершины $(a, i),(b, j)$ тогда и только тогда соединены ребром с меткой $x \in Z_{k}$, ведущим из $(a, i)$ в $(b, j)$, когда $i<t, j=i+1$, и $q_{x}(a)=b$.

Из определения 4.1 следует, что $t$-развертка является ациклическим графом и может содержать параллельные ребра.

Отметим ряд свойств $t$-развертки.

1. Множество вершин графа $R\left(t ; a_{0}\right)$ разбивается на $t+1$ непересекающихся подмножеств:

$$
V\left(t ; a_{0}\right)=\bigcup_{i=0}^{t} V_{i}\left(a_{0}\right)
$$

где $V_{i}\left(a_{0}\right)=\underset{a \in Q^{i}\left(a_{0}\right)}{\cup}(a, i)$ при $i \geq 0$.

2. Степень входа вершины $\left(a_{0}, 0\right)$ равна нулю. Степени входа остальных вершин графа $R\left(t ; a_{0}\right)$ положительны.

3. Степени выхода вершин, принадлежащих множеству $V_{t}\left(a_{0}\right)$, равны нулю. Степени выхода остальных вершин равны $k$.

4. Множество $E\left(t ; a_{0}\right)$ ребер графа $R\left(t ; a_{0}\right)$ разбивается на $t$ непересекающихся подмножеств:

$$
E\left(t ; a_{0}\right)=\cup_{i=1}^{t} E_{i}\left(a_{0}\right),
$$

где множество $E_{i}\left(a_{0}\right)$ состоит из ребер с концом в вершине из множества $V_{i}\left(a_{0}\right)$. Ребра, исходящие из одной вершины, имеют попарно разные метки.

5. $\left|V_{i}\left(a_{0}\right)\right|=\left|Q^{i}\left(a_{0}\right)\right|, i=0, \ldots, t$.

6. $\left|E_{i}\left(a_{0}\right)\right|=k \cdot\left|V_{i}\left(a_{0}\right)\right|, i=1, \ldots, t$. 
7. Любой путь максимальной длины (далее - максимальный путь) в графе $R\left(t ; a_{0}\right)$ имеет длину $t$.

Множество всех максимальных путей в графе $R\left(t ; a_{0}\right)$ обозначим $\Theta\left(t ; a_{0}\right)$.

Назовем $\Delta$-подграфом графа $R\left(t ; a_{0}\right)$ каждый его подграф $G$, являющийся объединением непустого множества путей $T \in \Theta\left(t ; a_{0}\right)$, причем ребра подграфа сохраняют присвоенные им метки.

Множество максимальных путей $\Delta$-подграфа $G$ обозначим $\Theta(G)$.

Так как положительные степени выхода вершин графа $R\left(t ; a_{0}\right)$ равны $k$, а положительные степени выхода вершин $\Delta$-подграфа $G$ меньше или равны $k$, то согласно определению 2.1 из [6] имеем $R\left(t ; a_{0}\right) \in B_{t}^{(k)}, G \in \Delta_{t}^{(k)}$.

4.2. Модуль $\boldsymbol{Z}\left(\boldsymbol{t}, \boldsymbol{a}_{\mathbf{0}}\right)$ дискретных функций. Аналогично [6] определим взаимно однозначное отображение $\Gamma: \Theta\left(t ; a_{0}\right) \rightarrow Z_{k}^{t}$, при котором пути $T \in \Theta\left(t ; a_{0}\right)$ соответствует $t$-последовательность $x^{(t)}=\left(x_{1}, \ldots, x_{t}\right)$ меток принадлежащих ему рёбер. Через $T\left(x_{1}, \ldots, x_{t}\right)$ обозначим путь $T \in \Theta\left(t ; a_{0}\right)$, для которого $\Gamma(T)=\left(x_{1}, \ldots, x_{t}\right)$.

Введем обозначения $\Phi_{t}$ для множества функций $g\left(x_{1}, \ldots, x_{t}\right): Z_{k}^{t} \rightarrow Z$, и $\Phi_{t}^{(k)} \subseteq \Phi_{t}$ для множества функций $g\left(x_{1} \ldots . x_{t}\right): Z_{k}^{t} \rightarrow Z_{k}$, называемых функциями $k$-значной логики [9].

Реберной функиией графа $R\left(t ; a_{0}\right)$, соответствующей ребру $e \in E\left(t ; a_{0}\right)$, назовём функцию $\varphi_{e}\left(x^{(t)}\right) \in \Phi_{t}$, задаваемую соотношением

$$
\varphi_{e}\left(x^{(t)}\right)=\left\{\begin{array}{l}
1, e \in \Gamma^{-1}\left(x^{(t)}\right), \\
0, e \notin \Gamma^{-1}\left(x^{(t)}\right) .
\end{array}\right.
$$

Заметим, что при $e \in E_{i}\left(a_{0}\right)$ функция $\varphi_{e}$ существенно зависит только от переменных из множества $x_{1}, \ldots, x_{i}$.

Множество всех линейных комбинаций реберных функций графа $R\left(t ; a_{0}\right)$ с коэффициентами из кольца $Z$ образует $Z$-модуль, который обозначим $Z\left(t ; a_{0}\right)$.

Применив теорему 2.1 из [6], получим

$$
\operatorname{dim} Z\left(t ; a_{0}\right)=1+(k-1) \cdot \sum_{i=0}^{t-1}\left|Q^{i}\left(a_{0}\right)\right| .
$$

Функции из множества $Z\left(t ; a_{0}\right)$ назовем $R$ - функциями. Функции из множества $Z\left(t ; a_{0}\right) \cap \Phi_{n}^{(k)}$ назовем $(R, k)$-функииями .

Каждая $R$-функция может быть задана коэффициентами $c_{e}^{f} \in Z$ линейной формы:

$$
f\left(x^{(t)}\right)=\sum_{e \in E\left(t ; a_{0}\right)} c_{e}^{f} \varphi_{e}\left(x^{(t)}\right),
$$

где $x^{(t)} \in Z_{k}^{t}$, причем такое задание не является единственным. 
Присвоим ребру $e \in E\left(t ; a_{0}\right)$ вес $c_{e}^{f}$. Весом $w(T, f)$ пути $T \in \Theta\left(t ; a_{0}\right)$ назовем сумму весов принадлежащих пути ребер. Из (4.1) следует соотношение $f\left(x^{(n)}\right)=w\left(\Gamma^{-1}\left(x^{(n)}\right), f\right)$. Следовательно, значение $w(T, f)$ не зависит от вида задания (4.1).

Два ребра графа $R\left(t ; a_{0}\right)$ назовем не сравнимыми, если не существует пути $T \in \Theta\left(t ; a_{0}\right)$, содержащего эти ребра.

Назовем $R$-функцию $f\left(x^{(n)}\right)$ ортогонально разложимой, если ее можно представить в виде

$$
f\left(x^{(n)}\right)=\sum_{e \in E} c_{e}^{f} \varphi_{e}\left(x^{(n)}\right),
$$

где $E$ - множество попарно не сравнимых ребер графа $R\left(t ; a_{0}\right)$.

Ортогонально разложимые $(R, k)$-функции назовём $(R, k$, о)-функциями.

Как следует из определения 4 и теорем 4.2, 4.3 статьи [7], если $f\left(x^{(n)}\right)$ является $(R, k, o)$-функцией, то для любого $b \in Z_{k}$ функция $f\left(x^{(n)}\right)+b$ также является $(R, k, o)$-функцией.

4.3. Граф решений $\boldsymbol{R}$-системы дискретных уравнений. Каждому непустому множеству $B \subseteq Z_{k}^{t}$ поставим в соответствие следующий $\Delta$-подграф графа $R\left(t ; a_{0}\right)$ :

$$
H(B)=\underset{b^{(t)} \in M}{\cup} \Gamma^{-1}\left(b^{(t)}\right) .
$$

Граф $H(B)$ может содержать максимальные пути, не принадлежащие множеству $B$. Выделяя случаи, когда этого не происходит, множество $B$ назовём $R$-замкнутым множеством, если $B=\Theta(H(B))$.

Назовем $R$-системой дискретных уравнений систему уравнений $S$, заданную соотношениями

$$
f_{j}\left(x^{(t)}\right)=a_{j}, \quad j=1, \ldots, m,
$$

если $f_{j}\left(x^{(t)}\right) \in Z\left(t ; a_{0}\right), a_{j} \in Z, j=1, \ldots, m$.

Графом решений $R$-системы уравнений $S$ назовём $\Delta$-подграф $G(S)$ графа $R\left(t ; a_{0}\right)$, удовлетворяющий условию $\Gamma(\Theta(G))=\operatorname{sol}(S)$.

Из принятых определений следует, что граф решений $R$-системы $S$ существует тогда и только тогда, когда система совместна и множество $\operatorname{sol}(S)$ является $R$-замкнутым.

Из теоремы 4.1 статьи [6] вытекает, что множество решений совместной однородной $R$-системы является $R$-замкнутым.

Систему уравнений $S$ назовём $(R, k, o)$-системой, если для $j=1, \ldots, m$ функции $f_{j}\left(x^{(t)}\right)$, являются $(R, k, o)$-функциями и $a_{j} \in Z_{k}$. 
Как показано в [7], из свойств ортогонально разложимых функций вытекает, что при произвольных значениях правых частей $a_{j} \in Z_{k}, j=1, \ldots, m$, однородная $(R, k, o)$-система $S^{\prime}$, заданная соотношениями

$$
f_{j}\left(x^{(t)}\right)-a_{k}=0, \quad j=1, \ldots, m,
$$

равносильна $(R, k, o)$-системе $S$, заданной соотношениями (4.2).

Трудоемкость метода решения $(R, k, o)$-системы $S$, заданной соотношениями (4.2), использующего переход к однородной $R$-системе, заданной соотношениями (4.3) с последующим применением алгоритма построения графа решений из [6], имеет порядок $m \sum_{i=0}^{t-1}\left|Q^{i}\left(a_{0}\right)\right|$ условных операций; такая же оценка справедлива для необходимого объема памяти.

Отметим, что предложенный в [8] вариант построения графа решений $(R, k, o)$-системы имеет те же оценки трудоемкости и объема памяти, но позволяет при наличии искажений в правой части находить те решения заведомо совместной $(R, k, o)$-системы, которые минимизируют ее невязку.

4.4. Определение входа инициального автомата по известному выходу. Рассмотрим стандартную для криптографического анализа задачу: по известной выходной последовательности (3.4) автомата $\boldsymbol{A}\left(a_{0}\right)$ перечислить все возможные входные последовательности.

Для $i=1, \ldots, t$ определим отображения $h_{i}(e): E_{i}\left(a_{0}\right) \rightarrow Z_{k}$, положив $h_{i}(e)=$ $=h(a, x)$, если ребро $е$ исходит из вершины $a \in V_{i-1}\left(a_{0}\right)$ и имеет метку $x$.

Определим функции $g_{i}\left(x^{(n)}\right), i=1, \ldots, t$, положив

$$
g_{i}\left(x^{(n)}\right)=\sum_{e \in E_{i}\left(a_{0}\right)} h_{i}(e) \cdot \varphi_{e}\left(x^{(n)}\right) .
$$

Так как ребра графа $R\left(t ; a_{0}\right)$, принадлежащие множеству $E_{i}\left(a_{0}\right)$, попарно не сравнимы, функции (4.4) являются $(R, k, o)$-функциями. В этом случае для любого $y \in Z_{k}$ имеем

$$
y=\sum_{e \in E_{i}\left(a_{0}\right)} y \varphi_{e}\left(x^{(n)}\right),
$$

откуда получаем

$$
g_{i}\left(x^{(n)}\right) \frac{-}{k} y=\sum_{e \in E_{i}\left(a_{0}\right)}\left(h_{i}(e)-y\right) \cdot \varphi_{e}\left(x^{(n)}\right),
$$

следовательно, $g_{i}\left(x^{(n)}\right)-y \in Z\left(t, a_{0}\right) \cap \Phi_{n}^{(k)}$.

Последовательности (3.4) соответствует следующая $(R, k, o)$-система уравнений $S(\boldsymbol{A})$, связывающая вход и выход автомата:

$$
\sum_{e \in E_{i}\left(a_{0}\right)}\left(h_{i}(e)-y_{k}\right) \cdot \varphi_{e}\left(x^{(n)}\right)=0, i=1, \ldots, t
$$


Следовательно, существует граф решений совместной системы $S(\boldsymbol{A})$, для построения которого можно использовать алгоритмы из [6] или [8].

4.5. Прямой алгоритм построения графа решений системы $S(A)$. В случае отсутствия искажений можно предложить более эффективный, чем упомянутые выше, алгоритм построения графа решений, использующий специфику системы $S(\boldsymbol{A})$, а именно - ее трапецеидальный вид.

Алгоритм 4.1. Исходные данные: выходная последовательность (3.4) автомата $\boldsymbol{A}\left(a_{0}\right)$.

Предварительный этап. Каждому ребру $e \in E_{i}\left(a_{0}\right)$ графа $R\left(t ; a_{0}\right)$, $i=1, \ldots, t$ присвоим вес $h_{i}(e)$.

I. Прямой ход алгоритма.

Шаг 0. Полагаем $V_{\mathrm{I}}^{(0)}=\left\{\left(a_{0}, 0\right)\right\}$.

Шаг 1 . Образуем и запоминаем множество $E_{\mathrm{I}}^{(1)}$ ребер графа $R\left(t ; a_{0}\right)$ с весом $y_{1}$, исходящих из вершины $\left(a_{0}, 0\right)$, и множество $V_{\mathrm{I}}^{(1)}$ вершин графа $R\left(t ; a_{0}\right)$, являющихся концами ребер из множества $E_{\mathrm{I}}^{(1)}$.

Если $E_{\mathrm{I}}^{(1)}=\varnothing$, то система несовместна. Если $E_{\mathrm{I}}^{(1)} \neq \varnothing$, то переходим к шагу 2.

Шаг $j, j=2, \ldots, t-1$. Образуем и запоминаем множество $E_{\mathrm{I}}^{(j)}$ ребер графа $R\left(t ; a_{0}\right)$ с весом $y_{j}$, исходящих из вершин множества $V_{\mathrm{I}}^{(j-1)}$, и множество $V_{\mathrm{I}}^{(j)}$ вершин графа $R\left(t ; a_{0}\right)$, являющихся концами ребер из множества $E_{\mathrm{I}}^{(j)}$. Если $E_{\mathrm{I}}^{(j)}=\varnothing$, то система несовместна. Если $E_{\mathrm{I}}^{(j)} \neq \varnothing$, то переходим к шагу $j+1$.

Шаг $t$. Образуем и запоминаем множество $E_{\mathrm{I}}^{(t)}$ ребер графа $R\left(t ; a_{0}\right)$ с весом $y_{t}$, исходящих из вершин множества $V_{\mathrm{I}}^{(t-1)}$, и множество $V_{\mathrm{I}}^{(t)}$ вершин графа $R\left(t ; a_{0}\right)$, являющихся концами ребер из множества $E_{\mathrm{I}}^{(t)}$. Если $E_{\mathrm{I}}^{(t)}=\varnothing$, то система несовместна. Если $E_{\mathrm{I}}^{(t)} \neq \varnothing$, то система совместна, и построен подграф $G_{\mathrm{I}}$ графа $R\left(t ; a_{0}\right)$ с множеством вершин $V\left(G_{\mathrm{I}}\right)=\bigcup_{j=0}^{t} V_{\mathrm{I}}^{(j)}$, и множеством ребер $E\left(G_{\mathrm{I}}\right)=\bigcup_{j=1}^{t} E_{I}^{(j)}$.

II. Обратный ход алгоритма.

Шаг 0. Полагаем $V^{(t)}=V_{\mathrm{I}}^{(t)}$.

Шаг 1. Удалив из множеств $V_{\mathrm{I}}^{(t-1)}, E_{\mathrm{I}}^{(t-1)}$ вершины с нулевой полустепенью выхода и инцидентные им ребра, получаем множества $V^{(t-1)}, E^{(t-1)}$.

Шаг $j, j=2, \ldots, t-1$. Удалив из множеств $V_{\mathrm{I}}^{(t-j)}, E_{\mathrm{I}}^{(t-j)}$ вершины с нулевой полустепенью исхода и инцидентные им ребра, получаем множества $V^{(t-j)}, E^{(t-j)}$.

Шаг $t$. Полагаем $V(0)=\left\{a_{0}\right\}$ 
В результате построен граф $G(S(A))$ с множеством вершин $V(G(S(A)))=$ $=\bigcup_{j=0}^{t} V^{(j)}\left(a_{0}\right)$ и множеством ребер $E(G(S(A)))=\bigcup_{j=1}^{t} E^{(j)}\left(a_{0}\right)$, являющийся графом решений системы $S(A)$.

В ходе реализации алгоритма необходимо запомнить граф $R\left(t ; a_{0}\right)$, метки и веса его ребер, а во время прямого и обратного хода алгоритма последовательно осуществлять преобразования непересекающихся подмножеств вершин и ребер графа $R\left(t ; a_{0}\right)$. Следовательно, трудоемкость $T(t)$ алгоритма 4.1 и необходимый объем памяти оцениваются величинами, имеющими порядок $O\left(V\left(R\left(t ; a_{0}\right)\right)\right)$.

4.6. Два примера. Как показано выше, трудоемкость построения графа решений $R$-системы дискретных уравнений линейно зависит от числа вершин $t$-развертки графа переходов автомата $\boldsymbol{A}\left(a_{0}\right)$. Следующие два примера приводятся для иллюстрации того факта, что число вершин $t$-развертки при $t \leq \rho(A)+\omega(A)-1$ может как экспоненциально (первый пример), так и полиномиально (второй пример) зависеть от $t$.

ПРИМЕР 4.1. Рассмотрим автомат

$$
\begin{gathered}
\boldsymbol{A}_{\mathbf{1}}\left(a_{0}^{(m)}\right)=\left(X, A_{1}, a_{0}^{(m)}, Y, q^{(1)}, h^{(1)}\right), \text { где } X=Y=Z_{k}, A_{1}=Z_{k}^{m}, \\
q_{x}^{(1)}\left(a_{1}, \ldots, a_{m}\right)=\left(a_{2}, \ldots, . a_{m}, x\right) \text { для }\left(a_{1}, \ldots, . a_{m}\right) \in A_{1}, x \in Z_{k}, \\
h^{(1)}\left(a^{(m)}, x\right): \operatorname{GF}(k)^{m} \rightarrow Z_{k} \text { - произвольная функция } k \text {-значной логики. }
\end{gathered}
$$

По входной последовательности $x_{i}, i=1, \ldots, t$, определяются состояния $a_{i}^{(m)}=q_{x_{i}}^{(1)}\left(a_{i-1}^{(m)}\right)$ автомата и вычисляются знаки выходной последовательности

$$
h^{(1)}\left(a_{i}^{(m)}\right)=y_{i}, \quad i=1,2, \ldots, t .
$$

Легко видеть, что автомат $\boldsymbol{A}_{1}$ является регулярным автоматом с одной компонентой сильной связности, поэтому $O\left(a_{0}^{(m)}\right)=A_{1}$.

При $k=2$ автомат $\boldsymbol{A}_{\mathbf{1}}$ совпадает с кодирующим устройством, рассмотренным в [10].

Положим $a_{0}^{(m)}=\left(b_{1}, \ldots, b_{m}\right) \in Z_{k}^{m}$.

Слоями автомата $\boldsymbol{A}_{\mathbf{1}}\left(a_{0}^{(m)}\right)$ являются следующие множества:

$$
\begin{gathered}
Q\left(a_{0}^{(m)}\right)=\left\{\left(b_{2}, \ldots, b_{m}, x_{1}\right), x_{1} \in Z_{k}\right\}, \\
Q^{2}\left(a_{0}^{(m)}\right)=\left\{\left(b_{3}, \ldots, b_{m}, x_{1}, x_{2}\right),\left(x_{1}, x_{2}\right) \in Z_{k}^{2}\right\}, \\
\left.\left.\ldots \ldots \ldots \ldots \ldots \ldots \ldots \ldots, \ldots \ldots, x_{m-1}\right) \in Z_{k}^{m-1}\right\}, \\
Q^{m-1}\left(a_{0}^{(m)}\right)=\left\{\left(b_{m}, x_{1}, \ldots, x_{m-1}\right),\left(x_{1}, \ldots, x,\right.\right. \\
Q^{m}\left(a_{0}^{(m)}\right)=A_{1} .
\end{gathered}
$$


Следовательно, $\rho\left(O\left(a_{0}^{(m)}\right)\right)=m-1, \omega\left(O\left(a_{0}^{(m)}\right)\right)=1,\left|Q^{j}\left(a_{0}^{(m)}\right)\right|=k^{j}$, $j=1, \ldots, m ;\left|V\left(R_{1}\left(t ; a_{0}^{(m)}\right)\right)\right|=\frac{k^{t+1}-1}{k-1}$.

Трудоемкость построения графа решений системы уравнений (4.5) алгоритмом 4.1 при $t \leq \rho(A)+\omega(A)-1$ имеет порядок $T_{1}(t)=O\left(k^{t}\right)$.

ПримЕР 4.2. Рассмотрим нелинейный автомат $\boldsymbol{A}_{\mathbf{2}}\left(a_{0}^{(m)}\right)$, построенный на основе регистра $U$ с линейной обратной связью над полем $\mathrm{GF}(p)$, где $p-$ простое число. Количество сдвигов регистра $U$ в каждом такте зависит от знака входной последовательности.

Примем обозначения:

$\boldsymbol{A}_{\mathbf{2}}\left(a_{0}^{(m)}\right)=\left(X^{(2)}, A_{2}, a_{0}^{(m)}, Y^{(2)}, q^{(2)}, h^{(2)}\right)$, где $X^{(2)}=Y^{(2)}=Z_{p}, A_{2}=\mathrm{GF}(p)^{m} ;$

$\boldsymbol{U}$ - матрица линейного преобразования максимального периода векторного пространства $\mathrm{GF}(p)^{m}$, реализуемого регистром $U$ автомата; для $a^{(m)} \in A_{2}, x \in Z_{p}$ имеем $q_{x}^{(2)}\left(a^{(m)}\right)=a^{(m)} \cdot \boldsymbol{U}^{x+1}$;

$h^{(2)}\left(a^{(m)}\right): \mathrm{GF}(p)^{m} \rightarrow Z_{p}-$ функция выхода автомата.

По входной последовательности $x_{i}, i=1, \ldots, t$, определяются состояния $a_{i}^{(m)}=a_{i-1}^{(m)} \cdot \boldsymbol{U}^{x+1}$ автомата и вычисляются знаки выходной последовательности

$$
h^{(2)}\left(a_{0}^{(m)} \cdot \boldsymbol{U}^{i+\sigma(i)}\right)=y_{i}, \quad i=1,2, \ldots, t .
$$

где $\sigma(i)=\sum_{j=1}^{i} x_{j}$

Легко видеть, что автомат $\boldsymbol{A}_{\mathbf{2}}$ является регулярным автоматом с двумя компонентами сильной связности: $\left\{0^{(m)}=(0, \ldots, 0)\right\}$ и $\left\{\mathrm{GF}(p)^{m} \backslash 0^{(m)}\right\}$. При $a_{0}^{(m)} \neq 0^{(m)}$ имеем $O\left(a_{0}^{(m)}\right)=\mathrm{GF}(p)^{m} \backslash 0^{(m)}$.

Слоями автомата $\boldsymbol{A}_{\mathbf{2}}\left(a_{0}^{(m)}\right), a_{0}^{(m)} \neq 0^{(m)}$, являются следующие множества:

$$
\begin{gathered}
Q\left(a_{0}^{(m)}\right)=\left\{a_{0}^{(m)} \cdot \boldsymbol{U}, \ldots, a_{0}^{(m)} \cdot \boldsymbol{U}^{p}\right\}, \\
\ldots \ldots \ldots \ldots \ldots \ldots \ldots . \\
Q^{i}\left(a_{0}^{(m)}\right)=\left\{a_{0}^{(m)} \cdot \boldsymbol{U}^{j} \mid i \leq j \leq i \cdot p\right\} .
\end{gathered}
$$

Легко убедиться в том, что:

$$
\begin{gathered}
\omega\left(O\left(a_{0}^{(m)}\right)\right)=1 ; \rho\left(O\left(a_{0}^{(m)}\right)\right)=\left[\frac{2^{n}-2}{p-1}\right] ; \\
\left.\left|Q^{i}\left(a_{0}^{(m)}\right)\right|=i(p-1)+1 \text { (при } i<\frac{2^{n}-2}{p-1}\right) ;
\end{gathered}
$$




$$
\begin{gathered}
\left.\left|Q^{i}\left(a_{0}^{(m)}\right)\right|=2^{n}-1 \text { (при } i \geq \frac{2^{n}-2}{p-1}\right) ; \\
\left.\left|V\left(R_{1}\left(t ; a_{0}^{(m)}\right)\right)\right|=(p-1) \frac{t(t+1)}{2}+t+1 \text { (при } i<\frac{2^{n}-2}{p-1}\right) .
\end{gathered}
$$

Трудоемкость построения графа решений системы уравнений (4.6) алгоритмом 4.1 при $t \leq \rho(A)+\omega(A)-1$ имеет порядок $T_{2}(t) \approx O\left(t^{2}\right)$.

\section{Список литературы}

1. Глухов М. М. О числовых параметрах, связанных с заданием конечных групп системами образующих элементов. - Труды по дискретной математике, т. 1, М.: ТВП, 1997, с. 43-66.

2. Лаллеман Ж. Полугруппы и комбинаторные приложения. - М.: Мир, 1985.

3. Ляпин Е. С. Полугруппы. - М.: ФИЗМАТЛИТ, 1960.

4. Клиффорд А., Престон Г. Алгебраическая теория полугрупп, т. 1. - М.: Мир, 1972.

5. Оре О. Теория графов. - М.: Наука, 1968.

6. Смирнов В.Г. Методы построения графов решений для систем $k$-значных уравнений. - Труды по дискретной математике, т. 8, М.: ФИЗМАТЛИТ, 2004, с. 253-280.

7. Смирнов В. Г. Методы решения систем дискретных уравнений, основанные на построении графа решений. - Труды по дискретной математике, т. 9, М.: Гелиос АРВ, 2006, с. 308-339.

8. Смирнов В. Г. Методы решения систем квазисимметрических уравнений и их применение к анализу генераторов с управляемым движением. Математические вопросы криптографии, 2010, т. 1, № 3, с. 67-91.

9. Яблонский С. В. Введение в дискретную математику. - М.: Наука, 1986.

10. Сумароков С. Н. Запреты двоичных функций и обратимость для одного класса шифрующих устройств. - Обозр. прикл. и промышл. матем., 1994, т. 1, вып. 1, с. 33-55. 

